\title{
Reshaping text data for efficient processing on Amazon EC2
}

\author{
Gabriela Turcu ${ }^{\mathrm{a}, *}$, Ian Foster ${ }^{\mathrm{b}}$ and Svetlozar Nestorov ${ }^{\mathrm{c}}$ \\ ${ }^{a}$ Computer Science Department, University of Chicago, Chicago, IL, USA \\ ${ }^{\mathrm{b}}$ Argonne National Laboratory, Computer Science Department, University of Chicago, Chicago, IL, USA \\ ${ }^{\mathrm{c}}$ Computation Institute, Chicago, IL, USA
}

\begin{abstract}
Text analysis tools are nowadays required to process increasingly large corpora which are often organized as small files (abstracts, news articles, etc.). Cloud computing offers a convenient, on-demand, pay-as-you-go computing environment for solving such problems. We investigate provisioning on the Amazon EC2 cloud from the user perspective, attempting to provide a scheduling strategy that is both timely and cost effective. We derive an execution plan using an empirically determined application performance model. A first goal of our performance measurements is to determine an optimal file size for our application to consume. Using the subset-sum first fit heuristic we reshape the input data by merging files in order to match as closely as possible the desired file size. This also speeds up the task of retrieving the results of our application, by having the output be less segmented. Using predictions of the performance of our application based on measurements on small data sets, we devise an execution plan that meets a user specified deadline while minimizing cost.
\end{abstract}

Keywords: Cloud computing, provisioning, Amazon EC2, text processing

\section{Introduction}

As the amount of available text information increases rapidly (online news articles, reviews, abstracts, etc.), text analysis applications need to process larger corpora. Increased computational resources are needed to support this analysis. Building and maintaining a cluster requires significant initial investments (hardware, physical space) and operational costs (power, cooling, management). Amortizing these costs demands maximizing resource utilization. However, optimizing for maximum utilization limits the ability of projects to grow their resource needs on short notice. Recently, commercially offered cloud computing [8] solutions (Amazon EC2, GoGrid, SimetriQ, Rackspace) have become an attractive alternative to inhouse clusters. They offer many advantages: customizable virtual machines, on-demand provisioning, usage based costs, fault tolerance. Some of the drawbacks are on the side of performance guarantees and security. Also, in a cluster environment, the user typically delegates the task of resource allocation to the local

\footnotetext{
*Corresponding author: Gabriela Turcu, Computer Science Department, University of Chicago, Chicago, IL 60637, USA. E-mail: gabri@cs.uchicago.edu.
}

resource manager, while the cloud user can take control of this step. We see this as an opportunity to steer application execution in such a way as to meet a user deadline while also minimizing costs.

A considerable amount of recent work has focused on analyzing the performance and cost effectiveness of such platforms for different classes of applications: CPU intensive or I/O intensive scientific computing applications $[6,10,11,20]$, service-oriented applications [7], latency-sensitive applications [2]. Other work has focused on quantifying the variation in received quality of service. Some of this work relies on simulations of a cloud environment, while most of it uses Amazon's Elastic Computing Cloud (EC2) as a testbed.

In this paper, we consider typical text processing applications (grep, part of speech tagging) and attempt to provide a good execution plan for them on Amazon EC2. Our input data sets consist of a large number of small files. We assume knowledge of the distribution of the file sizes in the input data set, and no knowledge of the internals of the application we are running. Our first goal is to quantify the potential performance loss suffered by our applications when consuming small files. To achieve this goal we observe the application's behavior on Amazon EC2 for different file sizes and identify a suitable file size or range 
of sizes. We then reshape our input data by grouping and concatenating files to match the preferred size as closely as possible. The text processing applications we consider do not need to be further modified to be capable to consume the concatenated larger input files. This approach will also imply a lower number of output files which results in a shorter retrieval time for the application results. This, in turn, results in a shorter makespan for the application. In terms of cost, the perbyte transferred cost being constant, the main benefit results from saved compute time due to the shorter makespan. Further, the cost savings are additive for applications which support multiple transactions.

A second goal of our work is to use our application as a benchmark on Amazon EC2 to determine a simple performance model for our application and use it to generate an execution plan for the entire input data. In order to devise a schedule we need estimates of the application runtime on Amazon resources. We observe the application's behavior on EC2 instances for small subsets of our data and then attempt to determine a predictor of runtime for larger subsets of our final workload. We consider linear, power law and exponential functions as predictors.

\subsection{Background}

The Elastic Computing Cloud (EC2) from Amazon offers its customers on-demand resizable computing capacity in the cloud with a pay-as-you-go pricing scheme. Amazon relies on Xen virtualization to offer its customers virtual hosts with different configurations. The user can request different instance types (small, medium, large) with different CPU, memory and I/O performance. The instance classification is based on the notion of an EC2 compute unit which is equivalent to a $1.0-1.2 \mathrm{GHz} 2007$ Opteron 2007 Xeon processor. The user can choose among a range of Amazon Machine Images (AMIs) with different configurations (32-bit/64-bit architecture, Fedora/Windows/Ubuntu). Users can modify AMIs to suit their needs and reuse and share these images.

Amazon allows the user to place an instance in one of the 3 completely independent EC2 regions (US-east, US-west, EU-west). This would allow the user to pick a location closer to where their data is available. Within a region, the users can choose to place their instances in different availability zones which are constructed by Amazon to be insulated from one another's failure. For example, the US-east region has 4 availability zones (us-east-1a, us-east-1b, us-east-1c and us-east- 1d). These zones are defined separately for each user. Amazon's SLA commitment is $99.95 \%$ availability for each Amazon EC2 region for every user.

Amazon instances come with ephemeral storage (160 GB for small instances). Amazon also offers the possibility to purchase Elastic Block Store (EBS) persistent storage. EBS storage volumes are exposed as raw block devices that can be attached to an instance and persist beyond the life of that instance. Multiple EBS storage volumes may be attached to the same instance, but an EBS storage volume may not be attached to multiple instances at the same time. The root partition of an instance may be of type instance-store in which case its contents are lost in case of a crash, or of type $e b s$ in which case its contents are persistent.

Amazon offers storage independent of EC2 via the Simple Storage Service (S3). Users can store an unlimited number of objects each of size of up to 5 GB. Multiple instances can access this storage in parallel with low latency, which is however higher and more variable than that for EBS storage volumes.

The pricing scheme for instances provides a flat rate for an hour or partial hour of computation $(\$ 0.1 \times\lceil h\rceil)$. This has implications for devising a good execution plan for an application. Once an instance is up and running, in most situations we will prefer to let it continue to run at least to the full hour.

Amazon has also started to offer spot instances. The price for these instances depends on current supply/demand conditions in the Amazon cloud. The user can specify a maximum amount she is willing to pay for a wall-clock hour of computation and configure her instance to execute whenever this maximum bid becomes higher than the current market offer. This is advantageous when time is less important of a consideration than cost. However, applications are required to be able to resume cleanly in order to best take advantage of spot instances. In our work, we are interested in being able to give cost effective execution plans when there are makespan constraints and so we use instances that can be acquired on demand.

\section{Motivation}

Our work is motivated by the computational needs of a project analyzing a large collection of online news articles. While the size of a single article is relatively small (a few dozen kilobytes), the total number of articles (tens of millions) and total volume of text (close to a terabyte) make the efficient processing of this data 
set challenging. In particular, we consider the idea of reshaping the original data, characterized by millions of small fragments with significant size differences, into large blocks of similar size. Processing these large blocks in parallel in the cloud is more attractive than dealing with the original data for two reasons. First, much of the overhead of starting many new instances and processes is avoided, making the overall processing more efficient. Second, the execution times for the similarly-sized blocks of data may also be relatively similar, thus enabling the estimation of the total running time and the optimization of the cost for typical pricing schemes given a deadline.

There are many other large collections of text that share the same characteristics as our target data set. For example, social scientists are interested in the evergrowing myriad of short texts generated by social network activities such as status updates, tweets, comments and reviews. Bioinformatics researchers often analyze a large number of abstracts, posters, slides, and full papers in order to extract new and emerging patterns of interactions among proteins, genes and diseases.

\section{Experimental setup}

In this section, we describe the resources we use on EC2 and the characteristics of our data sets.

\subsection{EC2 setup}

Small instances have been shown to be less stable [2, $7,21]$ but more cost effective for most applications. Our experiments use small instances since they are most common and most cost effective. We use a basic Amazon EC2 32-bit small instance running Fedora Core 8. Each such instance is configured with 1.7 GB memory, 1 EC2 compute unit, 160 GB local storage, 15 GB EBS root partition. The cost of an instance is $\$ 0.1$ per hour or partial hour. Payment is due only for the time when the instance is in the running state. Time spent starting up (pending state), shutting down (shutting down state) or in the terminated state is cost free.

We use the local instance storage for most of our experiments. Using EBS storage volumes, though adding to the cost of execution, has the advantage of simplifying how the execution plan would adapt to failure or bad performance. Although the virtualization layer promises uniform VM speed, our experience shows heterogeneity in instance performance. We observe in- stances behaving consistently slow or fast. Dejun et al. note in [7] CPU performance variability of up to a factor of 4 and significant I/O speed variations. If we decide an instance is not performing well, we may decide to let it run to the full hour while starting up another instance and attaching the EBS storage volume to it once it is ready. For an I/O intensive application, a simple calculation shows that if working with a slow instance with an average read speed of $60 \mathrm{MB} / \mathrm{s}$, we could process approximately $210 \mathrm{~GB}$ of data if we let the instance run for the next hour. If switching to another instance that is likely fast and consistent, even when paying a penalty of $3 \mathrm{~min}$ for the new instance startup and EBS storage volume attachment, we would still be able to process an extra 57 GB. If the instance happens to be slow we miss processing $10 \mathrm{~GB}$.

\subsection{Data}

We use two data sets in our experiments. The first is a set of HTML articles that are part of our Newslab collection. The Newslab data comprises of roughly 75 million news articles collected from Google News during the year of 2008. We use a subset of this data that corresponds to English language articles. This set comprises of approximately 18 million files adding up to a volume of almost $900 \mathrm{~GB}$. The majority of the files are less than $50 \mathrm{kB}$ and the distribution of the file sizes exhibits a long tail. The largest file size is $43 \mathrm{MB}$. Figure 1(a) shows the distribution up to files of size $300 \mathrm{kB}$. The file sizes are considered as multiples of $10 \mathrm{~K}$.

The second data set consists of 400,000 English language text files, extracted from a subset of HTML English language articles. The majority of the files are small $(<5 \mathrm{kB})$, while the largest file is $705 \mathrm{kB}$ in size. The plot below shows the frequency distribution of the sizes of the files up to $160 \mathrm{kB}$. The distribution has a long tail (Fig. 1(b)).

\section{Performance estimation}

Any execution strategy for an application on a set of resources relies on the expectation of how the application performs on each resources. Performance estimation can be done through analytical modeling [3,13], empirically [5] and by relying on historical data [17]. Since the characteristics of our cloud computing environment are volatile and opaque, we find that deter- 


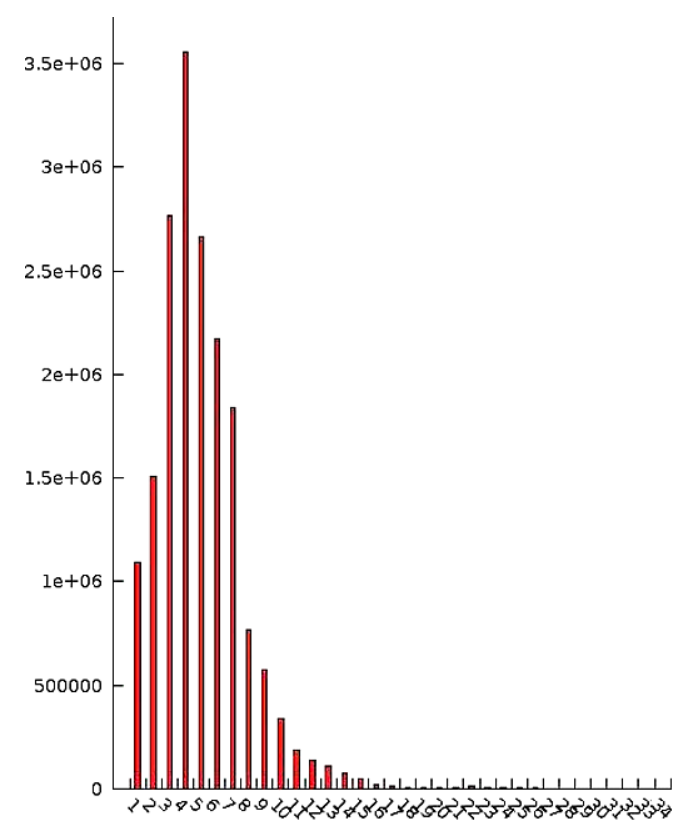

(a)

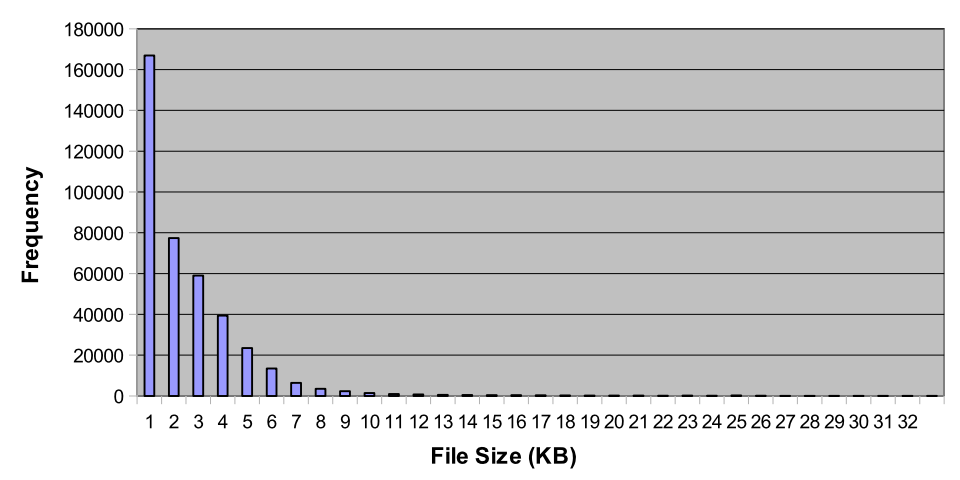

(b)

Fig. 1. Data sets. (a) Frequency distribution for the HTML data set HTML_18mil (10 kB bin). (b) Frequency distribution for the text data set Text_400K (1 kB bins). (Colors are visible in the online version of the article; http://dx.doi.org/10.3233/SPR-2011-0322.)

mining an empirical application performance model is preferable to using historical data or analytical models.

Our approach is to first acquire a stable, well performing instance. This is the case for most instances, but as noted before, it is possible to receive inconsistent instances or instances behaving consistently slow. Therefore, we first request a small instance and measure its performance using bonnie $++[1]$ to ensure that it is of high quality (over $60 \mathrm{MB} / \mathrm{s}$ block read/write performance). We repeat this performance measurement to confirm that the instance is stable. We repeat this procedure until we acquire an instance that performs well. The application performance model will be determined using this instance using a set of test probes constructed from our original data set. All performance measurements are repeated 5 times and the average and standard deviation are noted.

Next, we show how we derive test probes obtained by varying their construction along two dimensions: total volume size and base unit file size. The former is needed to understand the behavior of the application on larger inputs while the latter is needed to determine an appropriate file size for the application to consume. The procedure is described in detail below.

Our first probe consists of a single file. We designate it as probe $P_{\text {orig }}^{V_{0}}$, where $V_{0}$ represents the volume of data and the superscript notation indicates that the data is in its original form. We pick the initial file to send 
to be among the smallest in our data set. For every set of measurements on test probes, if the average execution time is small and the standard deviation is large, we continue to profile the application performance for larger volumes of data. For very short runs on small data sets, we note less stable measurements due to the domination of unstable setup overheads.

Next, varying on the volume dimension, we aim to construct probes of volume $V_{1}=k \times V_{0}$, where $k$ is chosen appropriately based on the amount of time taken to process the initial probe. We straightforwardly obtain probe $P_{\text {orig }}^{V_{1}}$ from our data set. Then we derive a further set of probes of the same volume $V_{1}$ by varying on the dimension of the unit file size. To achieve this, we use the first fit bin packing algorithm [19] to merge the original files into desired unit file sizes $\left(s_{0}, \ldots, s_{n}\right)$. We pick $s_{0}$ larger than the maximum file size in the original set. We then conveniently choose $s_{1}, \ldots, s_{n}$ as multiples of $s_{0}$, such that we perform the bin packing once to obtain $P_{s_{0}}^{V_{1}}$ and then directly derive the remaining probes $P_{s_{1}}^{V_{1}}, \ldots, P_{s_{n}}^{V_{1}}$. This approach is convenient since we avoid rerunning the first fit bin packing algorithm, but can be sensitive to the quality of the original bins of size $s_{0}$. We vary the unit file size up to the maximum possible size of $s_{n}=V_{1}$. We then analyze the performance of the original probe $P_{\text {orig }}^{V_{1}}$ and contrast it with the results for probes $P_{s_{0}}^{V_{0}}, \ldots, P_{s_{n}}^{V_{1}}$ in order to learn of any performance loss or gain that we would incur if the same data was organized in smaller or larger files.

If the results for the set of probes $\left(P_{\text {orig }}^{V_{1}}, P_{s_{0}}^{V_{1}}, P_{s_{1}}^{V_{1}}\right.$, $\left.\ldots, P_{s_{n}}^{V_{1}}\right)$ are not yet stable we continue this process with larger volumes. At the end of this process, we obtain measurements along three dimensions: data volume, unit file size and execution time.

Collecting the results for all the sets of probes we have, we can inspect each probe set to identify a possible preferable unit file size where the execution time is minimal. Sometimes we do not observe a single global minimum, but rather a plateau where the execution time is minimized. We give preference to choosing the preferred unit file size as the minimum from later probe sets that are more stable.

\section{Static provisioning}

Using the performance measurements collected, we can now proceed to build the application performance model. The performance measurement experiments al- low us to determine a single optimal unit file size or a range of unit file sizes which benefit overall application performance. Once a preferred unit file size is selected, we focus strictly on the measurements relevant to that unit file size. We perform regression to obtain a predictor for execution times as a function of data volume consumed. While this is a simple approach, we believe we can get a satisfactory estimate of the runtime without investing in determining complex performance models. Since our data points are not nearly equidistant, we perform the regression in logarithmic space. We consider the following models:

(1) Linear $y=a x$ : In logarithmic space, we would be fitting $Y=\ln a+X$, where $Y=\ln y ; X=$ $\ln x$.

(2) Power law $y=a x^{b}$ : In logarithmic space: $Y=$ $\ln a+b X$. We also fit functions of the form $Y=$ $a X^{2}+b X$ which correspond to original functions $y=x^{a \ln x+b}$.

(3) Exponential $y=a \mathrm{e}^{b x}$ : In logarithmic space: $Y=\ln a+b x$.

If we obtain a good fit through these means, we can use the predictor to estimate the total execution time required to process the entire data set.

Further, in constructing a provisioning plan, we make a few simplifying assumptions. Firstly, we assume all instances are uniform and performing well. As mentioned earlier, this is not the case in reality. In future work, we plan to extend our models to account for variability in instance performance. Secondly, we consider that for the grep application, the data is already staged onto EBS storage volumes and for the POS tagging application the data can be staged onto local storage in a constant time per run (assuming that the bottleneck is the maximum throughput available at the upload site).

Our goal is to determine the number of instances to be provisioned such as to process a volume $V$ of data while meeting a user set deadline $D$ and minimizing the cost of the computation. The pricing scheme considers a flat rate $r(0.085 \$$ for small instances $)$ for a full or partial hour of computation.

Then, for a predicted processing time $P$, a deadline $D$ and a linear predictor $y=a x$ :

- If $D \geqslant 1$, then the cost is $[P\rceil \times r$. If we ignore boot up time cost of the instances, then this would be equivalent to giving an hour's worth of computation for each instance and a partial hour to the last instance. This would also be the case if we pack $\lfloor D\rfloor$ hours of computation into each instance 


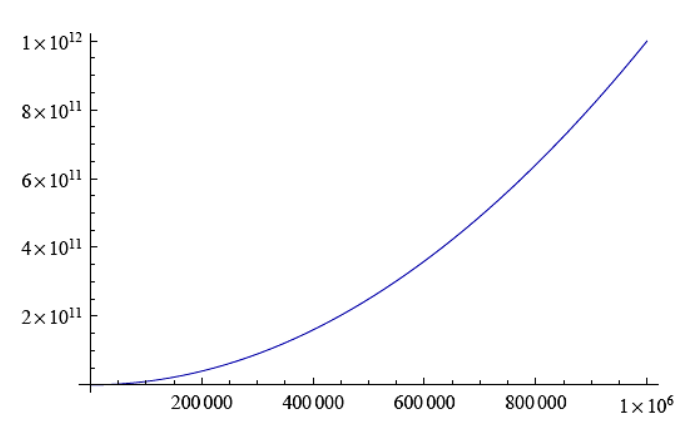

(a)

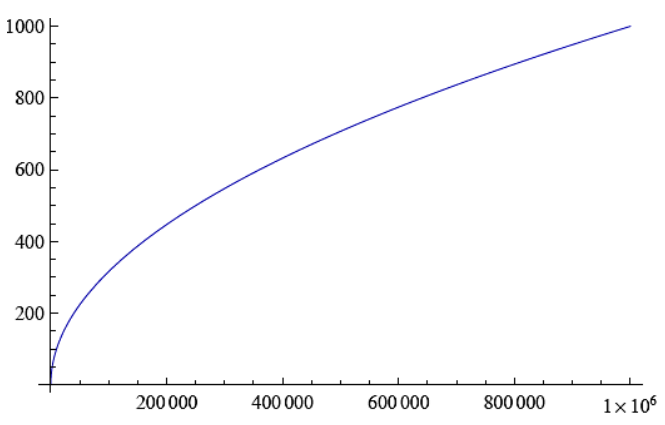

(b)

Fig. 2. Execution time as a function of data volume. (a) $f(x)=a x^{b}, a>0, b>1$. (b) $f(x)=a x^{b}, a>0, b<1$. (Colors are visible in the online version of the article; http://dx.doi.org/10.3233/SPR-2011-0322.)

(since the constant slope "a" ensures we process the same volume of data in either case).

- If $D<1, D>$ time taken to process largest (unsplittable) file, then the cost is $\left\lceil\frac{P}{D}\right\rceil \times r$, where we have no choice but to pay a full hour for instances running for time $D$

$$
f(d)= \begin{cases}r\lceil P\rceil, & d \geqslant 1 \\ r\left\lceil\frac{P}{d}\right\rceil, & d<1 .\end{cases}
$$

Further, we may repeat this process on non-overlapping subsets of the total volume of data. This would allow us to explore a larger volume of our data set through random sampling, at a smaller computational cost.

In general, we can improve our execution plan by considering more closely the performance models we derived. Figure 2 shows possible shapes for the fitted curves.

For $a>0, b>1\left(f^{\prime \prime}>0\right)$ (Fig. 2(a)), if startup time is small enough, it will always be better to start a new instance, since in a one-hour time slot we can process more data at smaller volumes than at larger volumes.

For $a>0, b<1\left(f^{\prime \prime}<0\right)$ (Fig. 2(b)), it will always be better to pack as much data as possible by $\lfloor D\rfloor$ than start a new instance. We will have to compare the volume of data that can be processed between times $\lfloor D\rfloor$ and $D$ to the volume that can be processed in $1 \mathrm{~h}$ from time 0 to 1 to decide which option is cheaper.

\subsection{Grep}

We run grep (GNU grep 2.5.1) on our first data set consisting of HTML files from the NewsLab data. Grep searches the files provided as input for matches of a provided pattern. The CPU-I/O mix of grep is heavily influenced by the complexity of the regular expression we are searching with and the number of matches found. Complex search patterns can tip the execution profile towards intense memory and CPU usage. Another factor is the size of the generated output which depends on the likelihood of finding a match and the size of the matched results.

We restrict ourselves to the usage scenario of searching for simple patterns consisting of English dictionary words. In our experiments we search for a nonsense word to increase as much as possible the likelihood that it is not found in the text. For a word that is not found we are sure to traverse all the data set regardless of other settings for grep, while also isolating from the cost incurred when also generating large outputs. We choose this full traversal worst case analysis since we believe it a processing pattern that occurs often in basic Natural Language Processing applications (e.g., tokenization).

We set our initial probe $P_{0}$ to a volume of $1 \mathrm{MB}$. Figure 3 shows the average execution times. We notice that the values are very small and the standard deviation over 5 measurements is large. We discard these results as too unstable and increase the volume of the probe.

We gradually increase the volume of the probe and unit file size. We notice that at the unit file size of $10 \mathrm{MB}$ we generally reach a plateau up to $2 \mathrm{~GB}$ (Fig. 4).

A more careful sampling of the file size unit range reveals that the plateau is not smooth as shown in Fig. 5. We observed spikes where the performance was degraded. The results are repeatable and stable in time, which rules out a contention state for networked storage. Our hypothesis is that, our probes, while on the same EBS logical storage volume, were placed in 


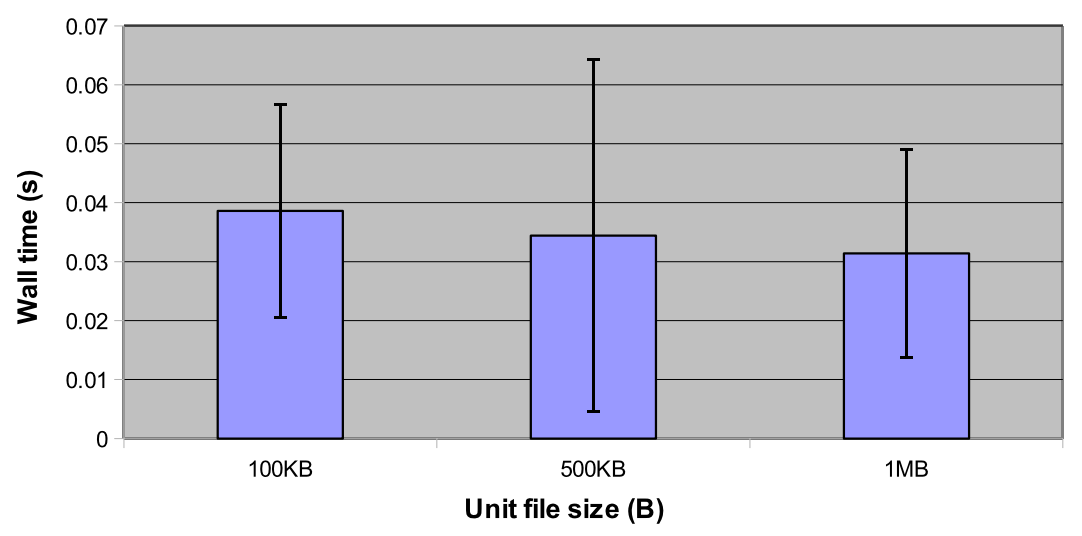

Fig. 3. Execution times for grep on a $1 \mathrm{MB}$ volume. (Colors are visible in the online version of the article; http://dx.doi.org/10.3233/SPR-20110322.)

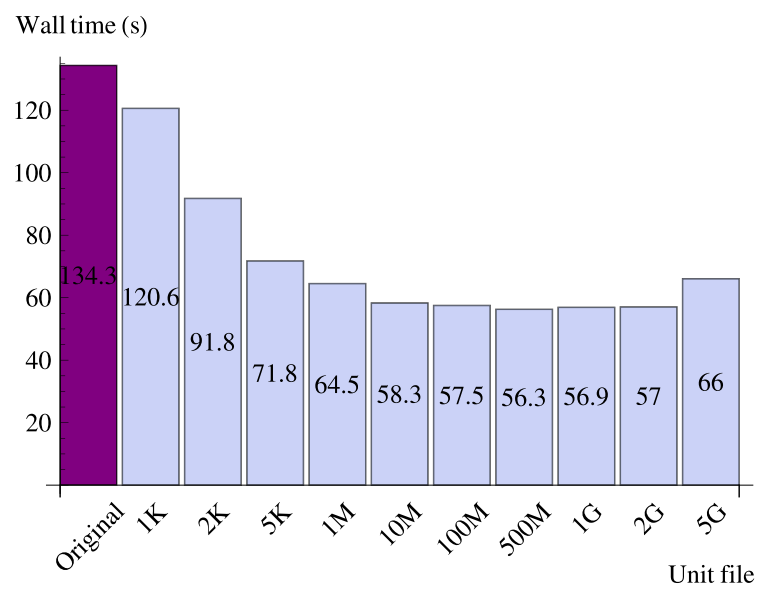

Fig. 4. Execution times for grep on a $5 \mathrm{~GB}$ volume. (Colors are visible in the online version of the article; http://dx.doi.org/10.3233/ SPR-2011-0322.)

different locations some of which have a consistently higher access time. We verified that this is indeed a possible cause by consistently observing that working with clones of a large sized directory can result in performance variations of up to a factor of 3 .

We select the unit file size to be $100 \mathrm{MB}$, which is in the minimum range and for which our experiments also have a small standard deviation. Based on the measurements we have already collected for this unit file size, we obtain a very good linear fit $\left(R^{2}=0.999\right.$ and very small residuals of magnitude $<1)$ :

$$
f(x)=-0.974+1.324 \times 10^{-8} x .
$$

We perform our experiments on a random $100 \mathrm{~GB}$ volume of the data set HTML_18mil and stage in this data equally across 100 EBS storage volumes. The deadline we wish to meet dictates how to attach the available volumes to the required number of instances. The unit of splitting of the data across the EBS storage volumes determines the coarseness of deadlines we can meet.

Let $V$ be the total volume of $100 \mathrm{~GB}, V^{0}=\frac{V}{100}$ be the data volume on each EBS device and $f^{-1}(D)=$ $V_{D}$ be the data volume that our model predicts can be processed by deadline $D$. If we consider a deadline $D<1$, if $V^{0}>V_{D}$, we can not directly meet this deadline without reorganizing our data to lower the unit volume $V^{0}$. If $V^{0}<V_{D}$, we can provide $\left\lfloor\frac{V_{D}}{V^{0}}\right\rfloor$ EBS devices each of volume $V^{0}$ to an instance. This would demand that we use $\left\lceil\frac{V}{\left[V_{D} / V^{0}\right] V^{0}}\right]=i$ instances. We can further improve the likelihood of meeting the deadline by balancing the volume across the $i$ instances or by lowering the deadline to be met and reevaluating the execution plan as described in the next section.

Based on our model given by Eq. (1), we predict that processing $100 \mathrm{~GB}$ of data within $D=3600 \mathrm{~s}$ only requires $1387.8 \mathrm{~s}$. The actual execution time is 1975.6 s. Figure 6 shows that we underestimate the deadline by almost $30 \%$. The figure also shows a 5.6 fold improvement on the execution time when working with $100 \mathrm{MB}$ files instead of the files in their original format of a few kilobytes in size.

A possible source of improvement for the predictive power of our performance model, is to consider random samples from our entire data set and reestimate our predictor. From our data set, we choose 10 random samples (without replacement) of $2 \mathrm{~GB}$ and measure the execution time of grep on these samples, and a few of their smaller subsets. We consider these sam- 


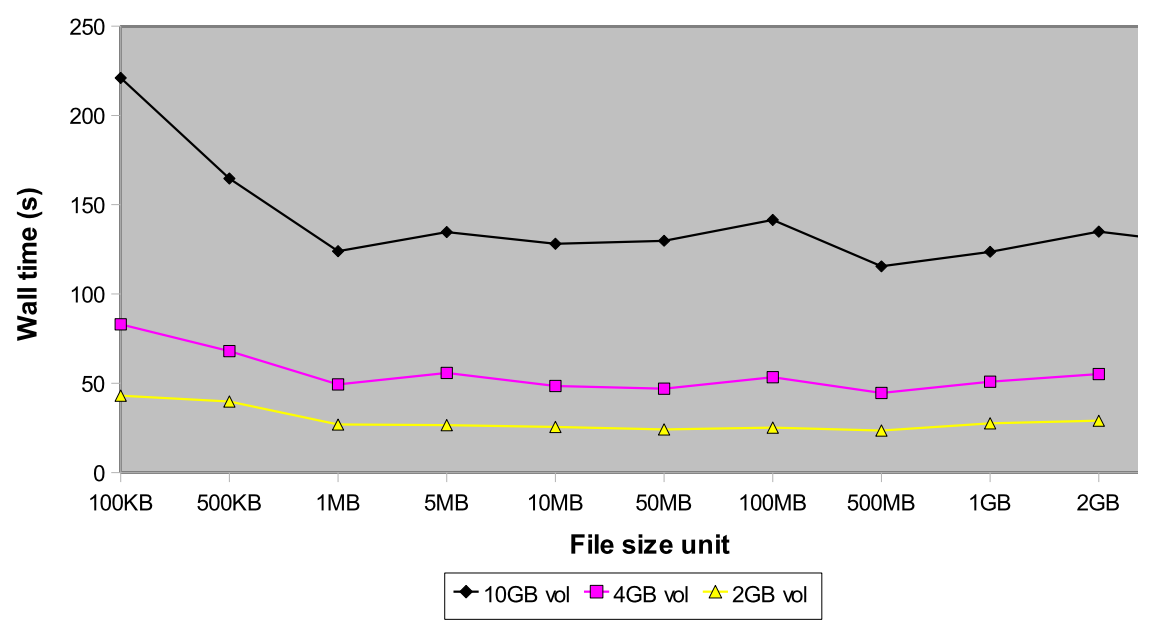

Fig. 5. Execution times for grep on 1, 2 and 10 GB volumes. (Colors are visible in the online version of the article; http://dx.doi.org/10.3233/ SPR-2011-0322.)

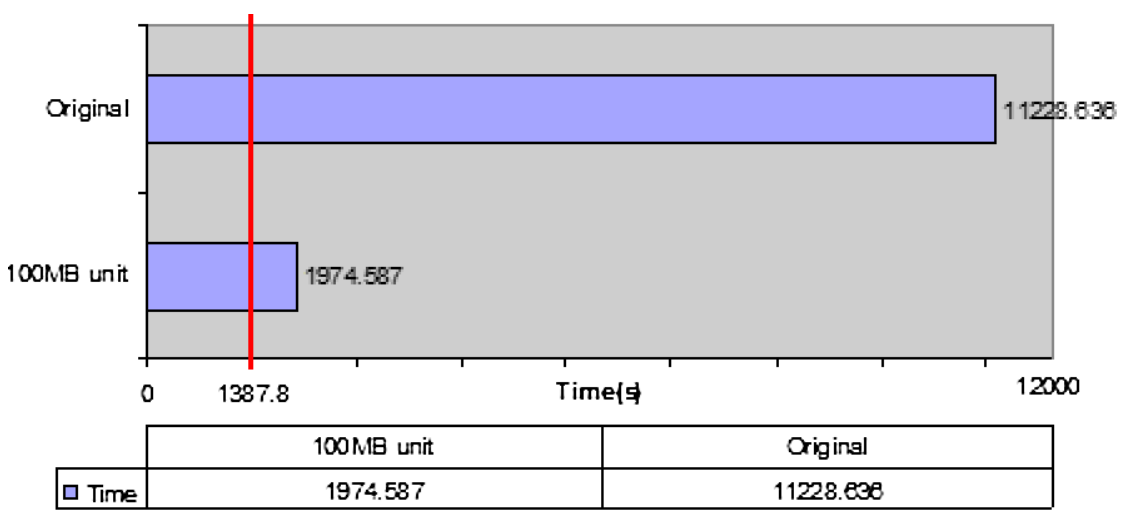

Fig. 6. Execution times for grep for $100 \mathrm{~GB}$. (Colors are visible in the online version of the article; http://dx.doi.org/10.3233/SPR-2011-0322.)

ples already in the chosen $100 \mathrm{MB}$ unit file size. The measurements show considerable variability: for the 10 samples, at the $2 \mathrm{~GB}$ volume, we obtain a minimum processing time of $23.25 \mathrm{~s}$, a maximum of $45.95 \mathrm{~s}$, average of $32.2 \mathrm{~s}$. We further refit our model to the new observations and obtain:

$$
f(x)=0.208+1.503 \times 10^{-8} x .
$$

The slightly higher slope of Eq. (2) improves the predicted execution time to $1576.44 \mathrm{~s}$, but this only reduces the error from $30 \%$ to $20 \%$ of the actual execution time.

\subsection{Stanford Part-of-Speech tagging}

The second application we consider is the Stanford Part-of-Speech tagger [18] which is a commonly used in computational linguistics as one of the first steps in text analysis. It is a Java application that parses a document into sentences and further relies on language models and context to assign a part of speech label to each word.

Our goal is to run the Stanford Part-of-Speech tagger with the left 3 words model on our second data set of 1 GB size. We wrap the default POS tagger class that is set up to parse a single document, such that we process a set of files avoiding the startup cost of a new JVM for every file.

We note that over $40 \%$ of our files are less than $1 \mathrm{kB}$ in size. Based on this, we pick the initial file size unit $s_{0}$ to be $1 \mathrm{kB}$, and let $V_{1}=1000 \mathrm{kB}$. Using the subset-sum first fit heuristic [19], we construct probe sets of volume $1000 \mathrm{~KB}$. The original probe contains over twice the number of files (2183) as the probe with unit file size of $1 \mathrm{kB}$ (1000). The average execution 


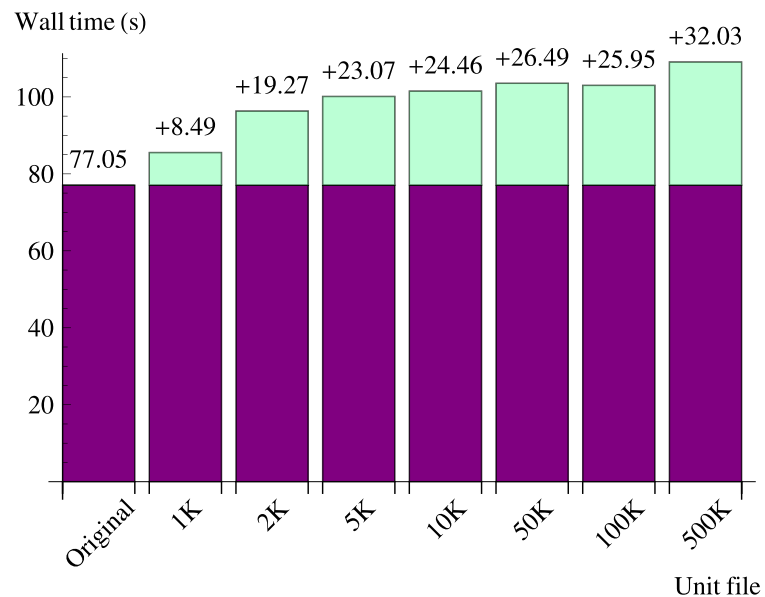

Fig. 7. Execution times for POS tagging on a volume of $1000 \mathrm{~K}$. (Colors are visible in the online version of the article; http://dx.doi.org/10.3233/SPR-2011-0322.)

time over 5 measurements for the probe set is shown in Fig. 7.

We observe that the original level of segmentation fairs the best and using a smaller number of larger files does not provide any benefits. The application is memory bound and does not benefit from dealing with larger file sizes.

Keeping the original level of segmentation for the files, we attempt a linear fit of form $f(x)=a x+b$ for our measurements. We obtain a good fit on which we base our predictions:

$$
f(x)=0.327+0.865 \times 10^{-4} x .
$$

Let the total volume of our data set be $V$, and the desired deadline be $D$. For a deadline of one hour ( $D=3600$ ), we solve Eq. (3) for $y=3600$ and obtain the solution $x_{0}$ which represents the amount of data that can be processed within the deadline according to our performance model. The solution prescribes we need $i_{0}=\left\lceil\frac{V}{\left\lfloor x_{0}\right\rfloor}\right\rceil=\lceil 26.1\rceil=27$ instances. We then proceed to pack our data set into 27 bins. For this step, we consider the input files in their original order. If we apply the first fit algorithm to the file sizes sorted in descending order, we are more likely to obtain bins that closely match the prescribed capacity. However, this will result in the first bins containing a small number of large files and the latter bins containing many small files. Our experiments for the POS application show that the degradation for working with large files is pronounced. Therefore, we consider the files in the order in which they are provided, though improvements are possible considering more refined information about the distribution of the file sizes. With this approach we obtain the result shown in Fig. 8(a).

We can improve our schedule, by uniformly distributing the data to each instance (Fig. 8(b)). In this way, we reduce the chance of missing the deadline, while still paying the same cost of $r \times i_{0}$. With the new bins of size $\frac{V}{i_{0}}$ we meet the deadline successfully.

For deadlines larger than $1 \mathrm{~h}$, if we consider performance prediction models that are linear, exponential or power law and that the instance start up time is insignificant, then the best strategy is to fit an hour of computation into as many instances as needed to complete the task. In reality, the instance startup times are not always insignificant and there are limitations on the number of instances that can be requested. For this reason, we want to find a schedule that also limits the number of instances requested.

When solving Eq. (3) for $D=7200$ and distributing the data uniformly to each instance, we obtain the results in Fig. 9(a). The deadline is met loosely.

A further improvement for our prediction can be obtained by taking random samples from our data set and reevaluating our performance model. To achieve this, we take 3 samples of $5 \mathrm{MB}$ each (without replacement) and measure the execution times for these samples and subsets of them. Including the new measurements, we obtain another linear fit of good quality:

$$
y=3.086+0.725482 \times 10^{-4} x .
$$

The slope of the new model is lower than that of the model in Eq. (3), indicating that for the same deadline, the new model will predict we can process more data. This matches the observation that based on the simple linear model from Eq. (3), we meet the deadline loosely enough that it may be possible that the deadline can be met with a lower number of instances.

Based on the new model in Eq. (4), we determine we require 22 instances for $D=3600$ (compared to the 27 determined by the earlier model) and 11 instances for $D=7200$ (compared to the 14 instances required by the earlier model). The results are shown in Figs 8(c) and $9(\mathrm{~b})$, respectively.

We note that the missed deadlines compensate for the benefit would have gotten by using a smaller number of instances. A reason for missing both deadlines when using the new model (given by Eq. (4)) was that we obtained very full bins, with little opportunity to distribute the data evenly across instances to a lesser volume (and correspondingly lesser deadline) than the 


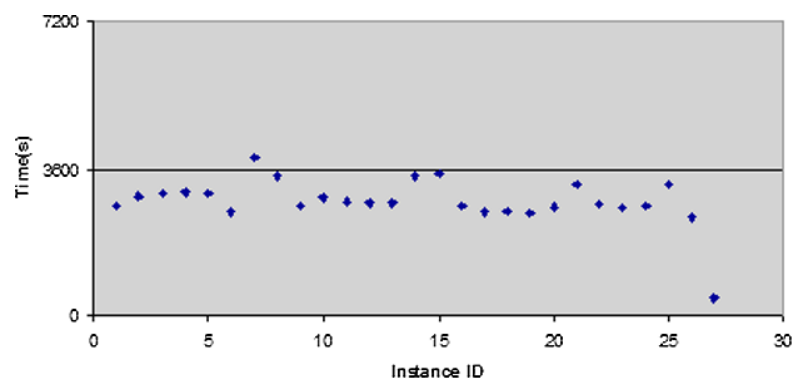

(a)

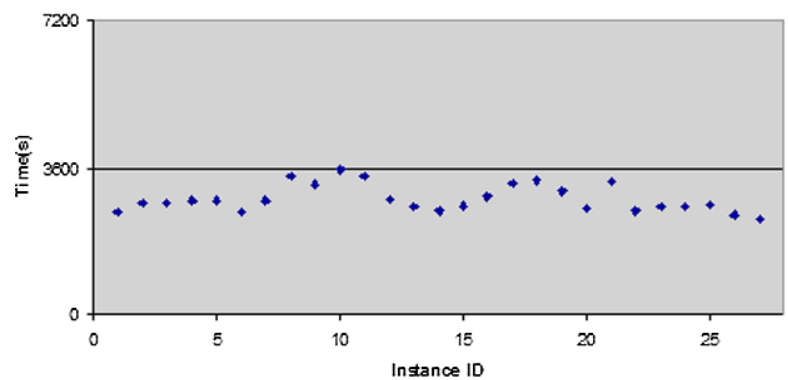

(c)

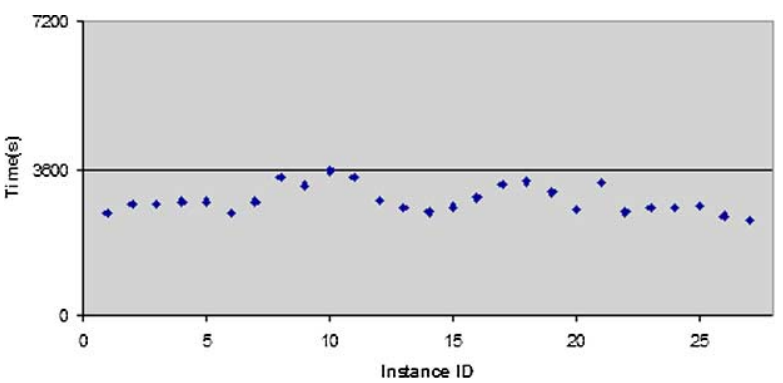

(b)

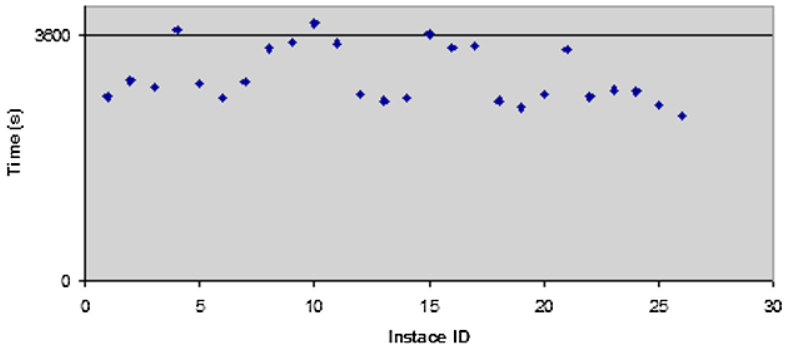

(d)

Fig. 8. POS execution times. (a) POS tagging for $D=1 \mathrm{~h}$, model (3). (b) POS tagging for $D=1 \mathrm{~h}$, uniform bins, model (3). (c) POS tagging for $D=1 \mathrm{~h}$, random sampling, model (4). (d) POS tagging scheduling for adjusted $D=3124$, model (4). (Colors are visible in the online version of the article; http://dx.doi.org/10.3233/SPR-2011-0322.)

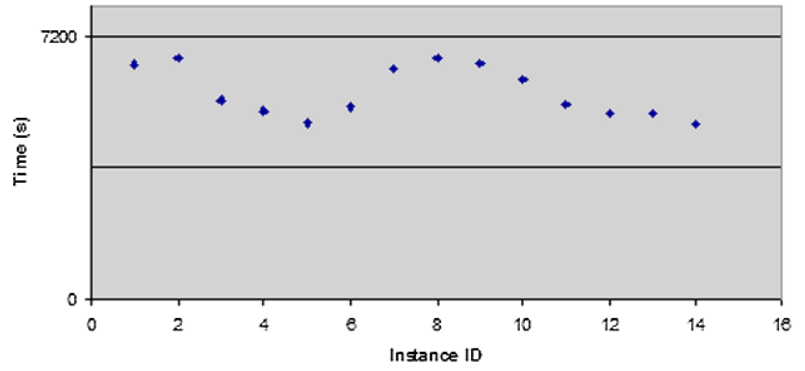

(a)

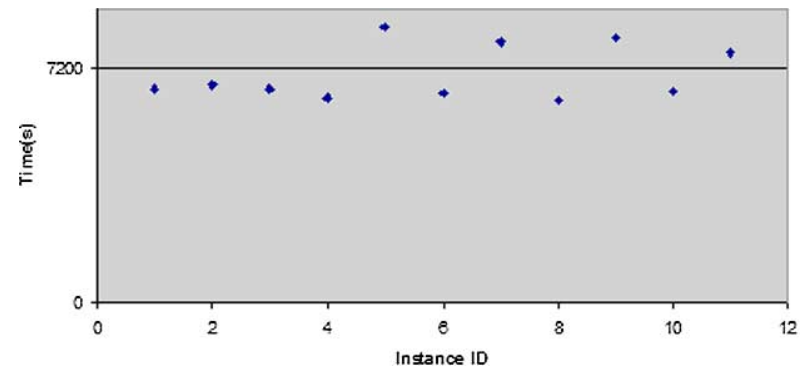

(b)

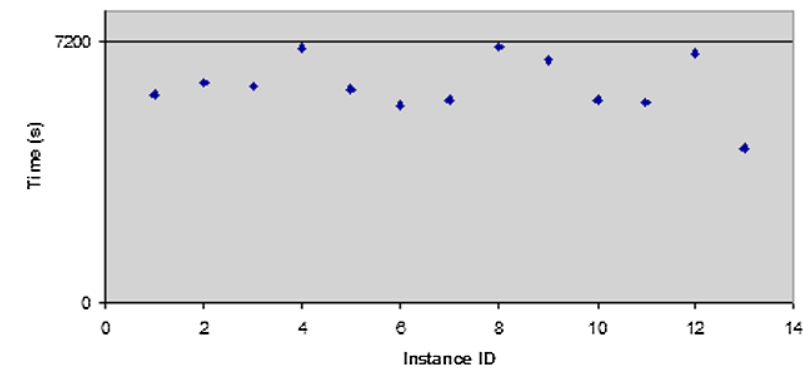

(c)

Fig. 9. POS execution times. (a) POS tagging scheduling for $D=2 \mathrm{~h}$, uniform bins, model (3). (b) POS tagging for $D=2 \mathrm{~h}$, random sampling, model (4). (c) POS tagging scheduling for adjusted $D=6247$, model (4). (Colors are visible in the online version of the article; http://dx.doi.org/10.3233/SPR-2011-0322.) 
one prescribed by $D$. When fitting with the earlier model (given by Eq. (3)) we happened to obtain the last bin relatively empty which permitted distributing the data uniformly over the instances at a smaller volume which then corresponds to a lower deadline than that which we must meet.

Based on the residuals for the model in (4), we consider it is acceptable to assume that the relative residuals $\frac{y-f(x)}{f(x)}$ are normally distributed. We would like to have a small probability of the residual at the predicted value exceeding some quantity. This can be translated in the value $y$ exceeding a deadline. Assume we would like to have a less than $10 \%$ chance to exceed a deadline: $P(y>D) \leqslant 0.1$. Or, in terms of the relative residual: $P\left(\frac{y-f(x)}{f(x)}>\frac{D-f(x)}{f(x)}\right) \leqslant 0.1$. Since the relative residual is assumed to be a normal random variable (call it $\left.X, P\left(X>\frac{D-f(x)}{f(x)}\right) \leqslant 0.1\right)$ can be standardized relying on the sample mean and sample standard deviation calculated from the residuals of our model $\mu_{X}$ and $\sigma_{X}$. Then, $P\left(Z>\frac{(D-f(x)) / f(x)-\mu_{X}}{\sigma_{X}}\right) \leqslant 0.1$, where if $P(Z>z) \leqslant 0.1$, gives $z=1.29$.

Then, $D=f(x)(1+a)$, where $a=1.29 \sigma_{X}+\mu_{X}$. For our residuals, we get $a=1.525$. This means, that in order to have a $10 \%$ chance of missing the deadline $D$, we need to choose $x$ such that $f(x)=\frac{D}{1+a}$. For $D=3600$, we should lower the deadline to $D 1=$ 3124 and for $D=7200$, we should lower the deadline to $D 1=6247$.

The results for the adjusted deadlines are given in Figs 8(d) and 9(c) respectively. The result for the original deadline of $1 \mathrm{~h}$, show that we miss the deadline fewer times than in Fig. 8(c), but pay for an equivalent 30 instance hours of computation, which happens to be a worse fit than when using the first model and consuming 27 instance hours only.

The results for the deadline of $2 \mathrm{~h}$ show that we are no longer missing the deadline and require 26 instance hours of computation. Without the adjusted deadline (Fig. 9(b)) we require the same number of instance hours, but miss the deadline. Both solutions are better than those predicted by the first linear model (Fig. 9(a)) which demands for 28 instance hours of computation.

Based on the calculation above, a good general strategy can be the following. For an initial deadline $D$, determine the minimum needed instances as $\left\lceil\frac{V}{V_{D}}\right\rceil=i$. If we are to distribute the data approximately uniformly over $i$ instances, we would give each at least $\left\lceil\frac{V}{i}\right\rceil=V_{D 1}$. The volume $V_{D 1}$ leads to $f\left(V_{D 1}\right)=D 1$. If the adjusted deadline that guarantees a $10 \%$ chance to miss $D$, i.e. $\frac{D}{1+a}$ is higher than $\mathrm{D} 1$, we are satisfied with distributing the data into $V_{D 1}$ bins over $i$ in- stances. Otherwise, we will schedule for the adjusted deadline $\frac{D}{1+a}$.

Another experiment highlights the performance variability of POS tagging for texts of similar size, but different language complexity. We choose the Dubliners novel by James Joyce and Agnes Grey by Emily Brönte available from the Gutenberg project [16]. While the difference in document size is less than 300 words (Dubliners - 67,496 words, Agnes Grey - 67,755 words), the POS analysis for the more complex text takes almost twice as long (Dubliners -6 min $32 \mathrm{~s}$, Agnes Grey -3 min $48 \mathrm{~s}$ ).

For our news data set, we do not see a dramatic improvement in the predictive power of our model derived by using random sampling. This can be expected of corpora that are uniform in terms of language complexity (average sentence length is an important parameter for POS tagging). For other corpora, as seen in the experiment above, random sampling can be vital to help capture the variation in text complexity.

\section{Related work}

A considerable amount of recent work focuses on investigating different aspects of commercial clouds: the quality of service received by users, the performance stability of the environment, the performancecosts tradeoffs of running different classes of applications in the cloud.

Walker [20] and Hazelhurst [10] investigate the effectiveness of constructing virtual clusters from Amazon EC2 instances for high-performance computing. Walker [20] relies on standard HPC benchmarks that are CPU intensive (NAS Parallel Benchmarks) or communication intensive (mpptest) to compare the performance of virtual clusters of EC2 instances to a real HPC cluster. Hazelhurst [10] performs a similar comparison using a real life memory and CPU intensive bioinformatics application (wcd). Both authors conclude that large EC2 instances fair well for CPU intensive tasks and suffer performance losses for MPI jobs that involve much communication over less efficient interconnects.

There is a lot of work that evaluates Amazon S3's $[9,15]$ performance and cost effectiveness for storing application data. However, there is less literature on the usage and performance of EBS storage volumes for large scale applications [4].

Deelman et al. [6] consider the I/O-bound Montage astronomy application and uses simulation to assess 
the cost vs performance tradeoffs of different execution and resource provisioning plans. One of the goals of their work is to answer a question similar to ours by finding the best number of provisioned instances and storage schemes to obtain a cost effective schedule. Their simulations do not take into account the performance differences among different instances and Amazon's flat hourly rate pricing scheme which discourages having an excessively large number of instances that run for partial hours.

Other work by Juve et al. [11] builds on [6] to address the more general question of running scientific workflow applications on EC2. They consider Montage as an I/O intensive application, and two other applications that are memory bound and CPU bound respectively and contrast the performance and costs of running them in the cloud with running on a typical HPC system with or without using a high performance parallel file system (Lustre). They note that I/O bound applications suffer from the absence of a high performance parallel file system, while memory-intensive and CPU-intensive applications exhibit similar performance. Their experiments are isolated to a single EC2 instance.

Wang and $\mathrm{Ng}$ [21] note the effect of virtualization on network performance, especially when the virtual machines involved are small instances that only get at most $50 \%$ of the physical CPU. They conclude that processor sharing and virtualization cause large network throughput and delay variations that can impact many applications.

Dejun et al. [7] analyze the efficacy of using Amazon EC2 for service oriented applications that need to perform reliable resource provisioning in order to maintain user service level agreements. They find that small instances are relatively stable over time, but different instances can exhibit performance of up to 4 times from each other, which complicates provisioning.

Continuing research on scheduling under time and cost constraints [22], recent work [12,14] investigates auto-scaling of resources or scheduling bag-of-tasks under these constraints.

\section{Future work}

On the performance modeling side, we would like to explore the improvements of using more complex statistics tools to improve the accuracy of our predictions. To account for the larger standard deviation of measurements at small data volumes, we can build a performance model using weighted curve fitting demanding closer fits in the large data volume range and allowing for looser fits in the small data volume range.

A further improvement can be made by tracking the quality of newly acquired instances and including instance quality likelihood estimates when devising an execution plan. For applications that use local storage, we may decide to invest in lightweight tests to establish the quality of the instances and then use different predictors for each instance quality level to decide how much data to send to meet the deadline.

We can also monitor application performance during execution and make dynamic scheduling decisions. If we find unresponsive instances, we force their termination and reassign their task to another instance. If we find that the application performance is not satisfactory, depending on the severity level, we can decide to terminate poor instances right away or to let them run up to close to a full hour and then reassign the remaining work to new or existing instances. Relying on the persistent nature of EBS storage volumes and their capability to quickly be attached/detached from compute units, replacing poorly performing instances can be done easily without explicit data transfers (instances created in the same zone as an EBS storage volume should have consistent read/write performance to that EBS volume) or loss of data.

A direction for our future research is also to devise good execution plans for more complex workflows arising in text processing. We can schedule such workflows while making sure we assign full hour subdeadlines to groups of tasks [22]. We plan to further explore data management possibilities for different classes of text applications we handle.

\section{References}

[1] Bonnie ++ , http://www.coker.com.au/bonnie++/.

[2] S. Barker and P. Shenoy, Empirical evaluation of latencysensitive application performance in the cloud, in: Proceedings of MMSys 2010 - the First ACM Multimedia Systems Conference, Scottsdale, AZ, USA, February 2010.

[3] J. Cao, D.J. Kerbyson, E. Papaefstathiou and G.R. Nudd, Performance modelling of parallel and distributed computing using pace1, in: Proc. IEEE Int. Performance Computing and Communications Conference, IPCCC-2000, Phoenix, AZ, USA, February 2000, pp. 485-492.

[4] D. Chiu and G. Agrawal, Evaluating caching and storage options on the Amazon web services cloud, in: Proc. 11th ACM/IEEE Int. Conference on Grid Computing (Grid 2010), Brussels, Belgium, 2010. 
[5] K. Cooper et al., New grid scheduling and rescheduling methods in the grads project, in: Proc. NSF Next Generation Software Workshop: International Parallel and Distributed Processing Symposium, Santa Fe, NM, USA, IEEE Computer Society Press, 2004, pp. 209-229.

[6] E. Deelman, G. Singh, M. Livny, B. Berriman and J. Good, The cost of doing science on the cloud: the montage example, in: SC'08: Proc. 2008 ACM/IEEE Conference on Supercomputing, IEEE Press, Piscataway, NJ, USA, 2008, pp. 1-12.

[7] J. Dejun, G. Pierre and C.-H. Chi, EC2 performance analysis for resource provisioning of service-oriented applications, in: Proc. 3rd Workshop on Non-Functional Properties and SLA Management in Service-Oriented Computing, Stockholm, Sweden, November 2009.

[8] I. T. Foster, Y. Zhao, I. Raicu and S. Lu, Cloud computing and grid computing 360-degree compared, CoRR, abs/0901.0131, 2009.

[9] S.L. Garfinkel, An evaluation of amazon's grid computing services: Ec2, s3 and sqs, Technical Report TR-08-07, Computer Science Group, Harvard University, 2008.

[10] S. Hazelhurst, Scientific computing using virtual highperformance computing: a case study using the amazon elastic computing cloud, in: SAICSIT'08: Proc. 2008 Annual Research Conference of the South African Institute of Computer Scientists and Information Technologists on IT Research in Developing Countries, ACM, New York, NY, USA, 2008, pp. 94 103.

[11] G. Juve, E. Deelman, K. Vahi, G. Mehta, B. Berriman, B.P. Berman and P. Maechling, Scientific workflow applications on amazon ec2, in: Workshop on Cloud-based Services and Applications in conjunction with 5th IEEE International Conference on e-Science (e-Science 2009), Oxford, UK, December 2009.

[12] M. Mao, J. Li and M. Humphrey, Cloud auto-scaling with deadline and budget constraints, in: Proc. 11th ACM/IEEE In- ternational Conference on Grid Computing (Grid 2010), Brussels, Belgium, October 2010.

[13] G.R. Nudd, D.J. Kerbyson, E. Papaefstathiou, S.C. Perry, J.S. Harper and D.V. Wilcox, Pace - a toolset for the performance prediction of parallel and distributed systems, Int. J. High Perform. Comput. Appl. 14(3) (2000), 228-251.

[14] A. Oprescu and T. Kielmann, Bag-of-tasks scheduling under budget constraints, in: Proc. 2nd IEEE Int. Conference Cloud Computing Technology and Science (CloudCom 2010), Indianapolis, IN, USA, November/December 2010.

[15] M.R. Palankar, A. Iamnitchi, M. Ripeanu and S. Garfinkel, Amazon s3 for science grids: a viable solution?, in: Proc. Int. Workshop on Data-Aware Distributed Computing, ACM, New York, NY, USA, 2008, pp. 55-64.

[16] Project gutenberg, http://www.gutenberg.org/.

[17] W. Smith, I.T. Foster and V.E. Taylor, Predicting application run times using historical information, in: IPPS/SPDP'98: Proc. Workshop on Job Scheduling Strategies for Parallel Processing, Springer, London, 1998, pp. 122-142.

[18] Stanford part-of-speech tagger, http://nlp.stanford.edu/ software/tagger.shtml.

[19] V. Vazirani, Introduction to Approximation Algorithms, Springer, Berlin, 2003.

[20] E. Walker, Benchmarking amazon ec2 for high-performance scientific computing, USENIX Login 33(5) (2008), 18-23.

[21] G. Wang and T.E. $\mathrm{Ng}$, The impact of virtualization on network performance of amazon ec 2 data center, in: Proc. 3rd Workshop on Non-Functional Properties and SLA Management in Service-Oriented Computing, San Diego, CA, USA, 2010.

[22] J. Yu, R. Buyya and C.K. Tham, Cost-based scheduling of scientific workflow application on utility grids, in: E-SCIENCE'05: Proc. First Int. Conference on e-Science and Grid Computing, IEEE Computer Society, Washington, DC, USA, 2005, pp. 140-147. 

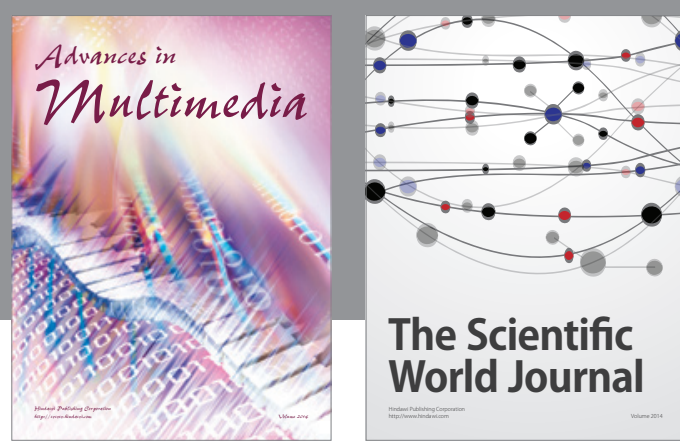

The Scientific World Journal
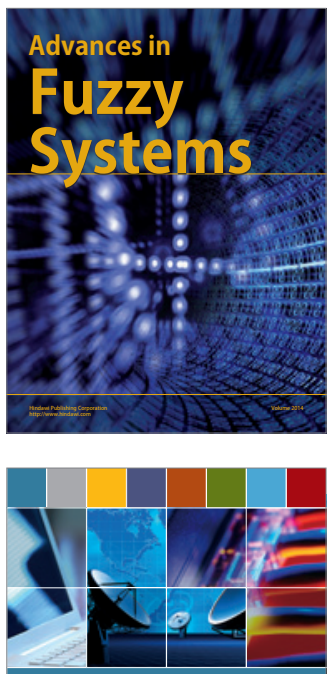

Computer Networks and Communications
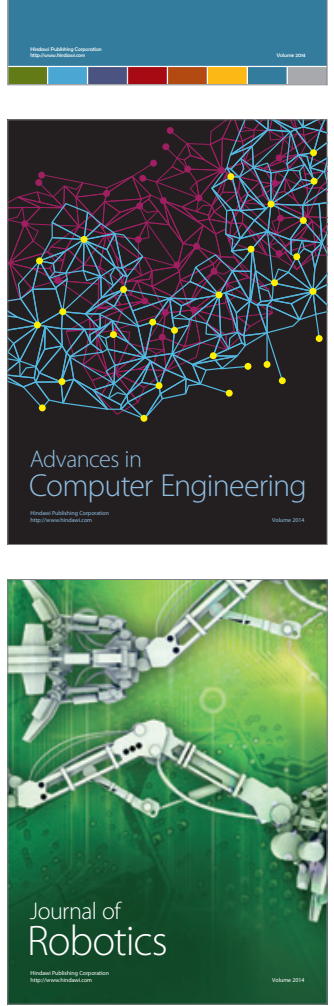
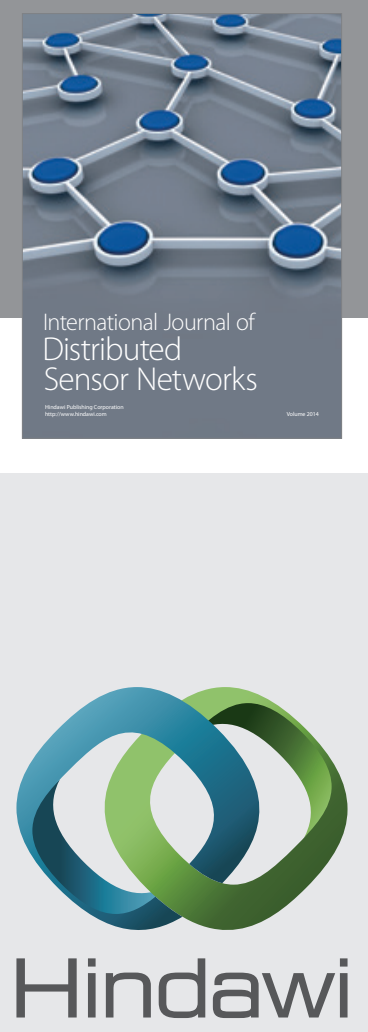

Submit your manuscripts at

http://www.hindawi.com
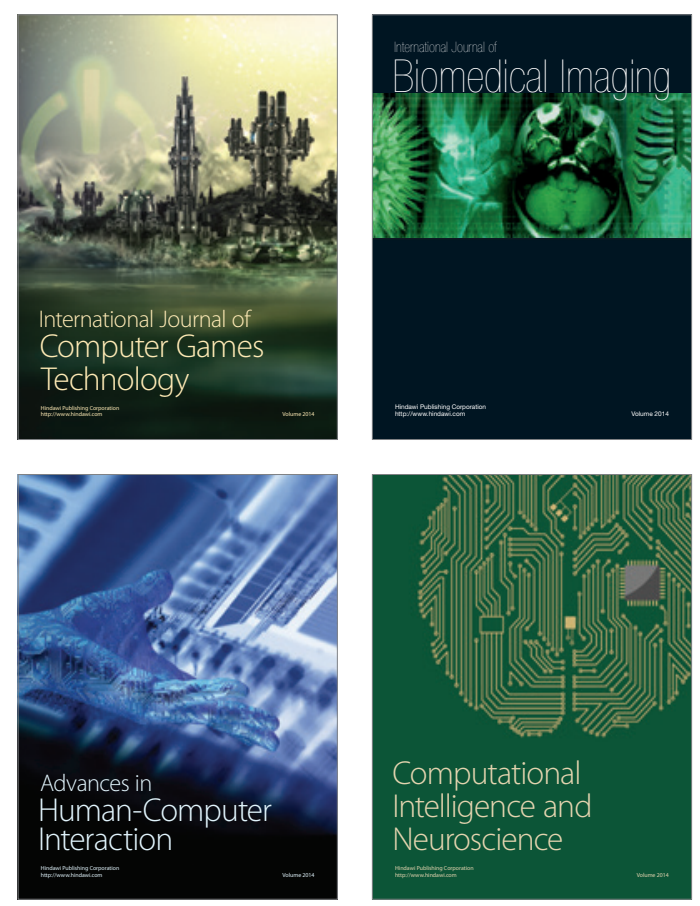
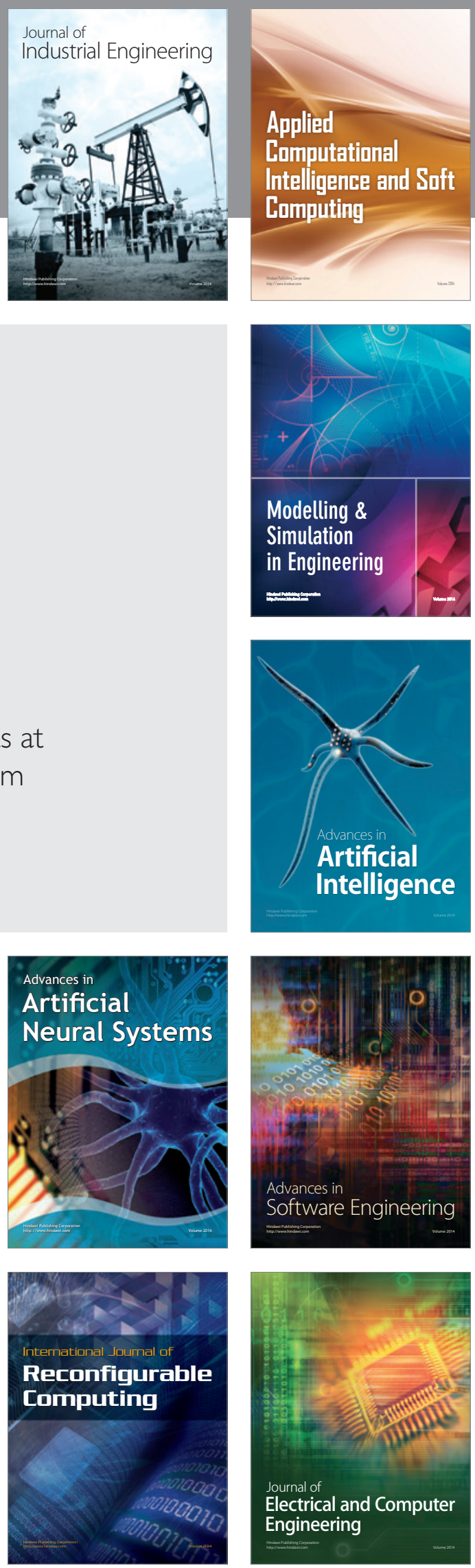